\title{
Fuzzy Comprehensive Evaluation of Research on China's Sports Industry Development in Leisure Era
}

\author{
Zhiyong $\mathrm{Xu}^{*}$
}

Department of Physical Education, Jiangsu Institute of Commerce, Nanjing 211168, Jiangsu, China

\begin{abstract}
In the leisure era, the sports industry has attracted more and more people's attention, and fitness centers became the main places of citizens' relaxation and entertainment in the city, which not only played roles in relaxation but also let sports fitness to gain well development. The paper utilizes fuzzy comprehensive evaluation to evaluate China's sports industrial development. The values obtain from comprehensive evaluation show that China's sports industrial contribution is good and shows an excellent development trend.
\end{abstract}

Keywords: Fuzzy evaluation, Leisure era, Physical health, Sports industry.

\section{INTRODUCTION}

Sports industry is China's important affiliated enterprise, and also an indispensible industry contributing to the state economy. China's sports industry's current situation is not going well, so in this paper we carried out status conditions analysis targeting at China's sports industry and conducted strategic researches on future development [1]. China's sports industry surely has many drawbacks, for example low starting points, imbalanced development and so on [2]. But the overall trend is increasing by year; economically developed cities have higher requirements on sports industry [3].

By Table 1, it is clear that rapidly economically developed cities also have very developed sports industry, which indicates that the sports industry can be regarded as the important pillar of economy, and also an important state industry [4]. Table 2 shows Hubei province sports industry's forms of ownership, which is dominated both by state-owned holding and private holding, and meanwhile Hubei province introduces proactive industries from Hong Kong, Macao and Taiwan, which not only introduces advanced enterprises, but also brings in rational management system and lets Hubei province sports industry to enter into a brand new developmental height [5].

Table 3 summarizes Hubei province's sports and relative industries investigation data.

Sports manufacturing industry occupies considerably large proportions in Hubei province sports industry, and it can be called as pillar industry. The sports manufacturing industry introduced gross value of production, covering sports sales, sports construction industry, and sports service industry. However, from Table $\mathbf{3}$, it is shown that Hubei province sports service industry development is not very impressive. However, if the sports industry gets rid of sports

*Address correspondence to this author at the Department of Physical Education, Jiangsu Institute of Commerce, Nanjing 211168, Jiangsu, China; Tel: +86-13584015148; E-mail: xuzhiyong2050@sina.com manufacturing industry, as shown in Fig. (1), it is established that sports sales and sports service industry will be increasing year by year, whereas the future of sports construction industry is surely uncertain so that it may have some connections with its attributes.

\section{FUZZY EVALUATION MODEL ESTABLISH- MENTS}

\subsection{Fuzzy Comprehensive Evaluation Model}

Fuzzy comprehensive evaluation model is suitable for fuzzy calculation of multiple factors that are uncertain. The paper utilizes fuzzy comprehensive evaluation with the following steps:

(1) At first, the paper establishes a factor set $U$ : $U=\left(\begin{array}{llll}U_{1} & U_{2} & \cdots & U_{k}\end{array}\right)$

(2) Secondly, it establishes a judgment set $V s$ (evaluation set).

(3) By establishing a fuzzy mapping from judgment matrix $U$ to judgment matrix $V$, it gets a fuzzy relation as the following matrix shows:

$$
R=\left[\begin{array}{cccc}
r_{11} & r_{12} & \cdots & r_{1 n} \\
r_{21} & r_{22} & \cdots & r_{2 n} \\
\vdots & \vdots & & \vdots \\
r_{m 1} & r_{m 2} & \cdots & r_{m n}
\end{array}\right]
$$

(4) By establishing a weight set, $A=\left(a_{1}, a_{2}, \cdots, a_{n}\right)$, the condition that should be met is :

$$
\sum_{i=1}^{n} a_{i}=1 \quad a_{i} \geq 0
$$

(5) In fuzzy relation $R$, every line reflects the line influencing factors to judgment-object extent, and 
Table 1. China's sports industry status analysis.

\begin{tabular}{|c|c|c|c|c|c|}
\hline Province & Guangdong & Jiangsu & Beijing & Liaoning & Zhejiang \\
\hline \hline Ratio & $24 \%$ & $15 \%$ & $30 \%$ & $13 \%$ & $15 \%$ \\
\hline
\end{tabular}

Table 2. Hubei province sports industry's forms of ownership.

\begin{tabular}{|c|c|c|}
\hline Forms of Ownership & Amount of Holdings & Percentage \\
\hline \hline State-owned holding & 268 & 14 \\
\hline Collective-owned holding & 195 & 6 \\
\hline Hong Kong, Macao and Taiwan-owned holding & 32 & 31 \\
\hline Other holdings & 658 & 1 \\
\hline Foreign enterprise holdings & 21 & 49 \\
\hline Private holding & 1987 & 2 \\
\hline
\end{tabular}

Table 3. Sports industries' all kinds of output indicators.

\begin{tabular}{|c|c|c|}
\hline Industry & Year 2006 & Year 2007 2008 \\
\hline \hline Sports manufacturing industry & 5516789 & 7544667 \\
\hline Sports sales industry & 13347 & 174227 \\
\hline Sports construction industry & 35347 & 62678 \\
\hline Sports service industry & 94674 & 105384 \\
\hline
\end{tabular}

meanwhile, $R$ in every column reflects the column influencing factors to judgment-object extent.

$$
\sum_{i=1}^{n} r_{i j} \quad j=1,2,3, \cdots, m
$$

Secondly, carry out following calculation:

$$
\begin{aligned}
B & =A \cdot R \\
& =\left(a_{1}, a_{2}, a_{3}, \cdots, a_{n}\right) \cdot\left[\begin{array}{cccc}
r_{11} & r_{12} & \cdots & r_{1 n} \\
r_{21} & r_{22} & \cdots & r_{2 n} \\
\vdots & \vdots & & \vdots \\
r_{m 1} & r_{m 2} & \cdots & r_{m n}
\end{array}\right] \\
& =\left(b_{1}, b_{2}, b_{3}, \cdots, b_{n}\right)
\end{aligned}
$$

In $V$, evaluation of fuzzy set $B$ describes the combination. Based on the above described facts, a simple modified model that is obtained by fuzzy comprehensive evaluation is shown as Fig. (1):

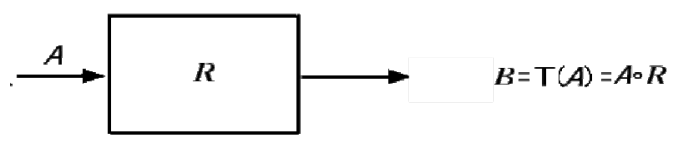

Fig. (1). Modified model.
According to Fig. (1), the modified fuzzy comprehensive evaluation model is established which corresponds to every factor of the grade evaluation transformation function, so that evaluation factors $\mathrm{u} 1, \mathrm{u} 2, \mathrm{u} 3, \mathrm{u} 4, \mathrm{u} 5$ membership functions can be expressed as the following formulas (1), (2), (3)..., (5) respectively:

$$
u_{v 1}\left(u_{1}\right)=\left\{\begin{array}{cc}
0.5\left(1+\frac{u_{i}-k_{1}}{u_{i}-k_{2}}\right), & u_{i} \geq k_{1} \\
0.5\left(1-\frac{k_{1}-u_{i}}{k_{1}-k_{2}}\right), & k_{2} \leq u_{i}<k_{1} \\
0 \quad, & u_{i}<k_{2}
\end{array}\right.
$$

$$
u_{v 2}\left(u_{1}\right)=\left\{\begin{array}{lr}
0.5\left(1-\frac{u_{i}-k_{1}}{u_{i}-k_{2}}\right), & u_{i} \geq k_{1} \\
0.5\left(1+\frac{k_{1}-u_{i}}{k_{1}-k_{2}}\right), & k_{2} \leq u_{i}<k_{1} \\
0.5\left(1-\frac{u_{i}-k_{3}}{k_{2}-k_{3}}\right), & k_{3} \leq u_{i}<k_{2} \\
0.5\left(1-\frac{k_{3}-u_{i}}{k_{2}-u_{i}}\right), & u_{i}<k_{3}
\end{array}\right.
$$


Table 4. China's sports undertaking evaluation indicator system.

\begin{tabular}{|c|c|c|c|}
\hline Sports construction industry $U_{1}$ & Sports personnel cultivation $U_{2}$ & Sports organizations cultivation $U_{3}$ & $\begin{array}{c}\text { Sports undertaking revitalization } \\
U_{4}\end{array}$ \\
\hline Sports facilities introduction $u_{11}$ & $\begin{array}{c}\text { Professional coaches cultivation } \\
u_{21}\end{array}$ & Sports events $u_{31}$ & $\begin{array}{c}\text { International sports undertaking } \\
\text { introduction } u_{41}\end{array}$ \\
\hline Sports facilities maintenance $u_{12}$ & Universities faculty cultivation $u_{22}$ & Sports activities $u_{32}$ & $\begin{array}{r}\text { Sports public undertaking develop- } \\
\text { ment } u_{42}\end{array}$ \\
\hline Stadium construction $u_{13}$ & $\begin{array}{c}\text { Sports foreign teachers introduction } \\
u_{23}\end{array}$ & Sports lecturing $u_{33}$ & $\begin{array}{r}\text { Revitalization of sports traditional } \\
\text { undertaking } u_{43}\end{array}$ \\
\hline $\begin{array}{c}\text { Daily sports facilities construction } \\
u_{14}\end{array}$ & Training strength $u_{24}$ & Sports overseas tour $u_{34}$ & \\
\hline $\begin{array}{c}\text { Sports equipment maintenance and } \\
\text { changing } u_{15}\end{array}$ & & & \\
\hline
\end{tabular}

Table 5. Four kinds of factors statistics ranking based on importance degree.

\begin{tabular}{|c|c|c|c|c|}
\hline Classification & Rank1 & Rank 2 & Rank 3 & Rank 4 \\
\hline \hline $\begin{array}{c}\text { Sports construction industry } \\
U_{1}\end{array}$ & 33 & 7 & 15 & 28 \\
\hline $\begin{array}{c}\text { Sports personnel cultivation } \\
U_{2}\end{array}$ & 0 & 0 & 23 & 12 \\
\hline $\begin{array}{c}\text { Sports organizations culti- } \\
\text { vation } U_{3}\end{array}$ & 0 & 9 & 19 & 21 \\
\hline $\begin{array}{c}\text { Sports undertaking revitali- } \\
\text { zation } U_{4}\end{array}$ & 3 & 3 & & \\
\hline
\end{tabular}

$$
u_{v 1}\left(u_{1}\right)=\left\{\begin{array}{cc}
0, & u_{i} \geq k_{2} \\
0.5\left(1-\frac{k_{1}-u_{i}}{k_{2}-k_{3}}\right), & k_{3} \leq u_{i}<k_{2} \\
0.5\left(1+\frac{k_{3}-u_{i}}{k_{2}-u_{i}}\right), & u_{i}<k_{3}
\end{array}\right.
$$

\subsection{Combine with Fuzzy Evaluation Models to Evaluate China's Sports Undertaking System}

By the above model principles, a factor set $U$ has been established, from which $U=\left(\begin{array}{llll}U_{1} & U_{2} & U_{3} & U_{4}\end{array}\right)$ is obtained. Among them, sports construction industry is $U_{1}$, sports personnel cultivation is $U_{2}$, sports organizations cultivation is $U_{3}$, and sports undertaking revitalization is $U_{4}$, see Table 4 . The paper establishes a small factor set out of four important factors sets.

Table 4 listed factors helped get an evaluation set.

$$
U_{1}=\left\{u_{11}, u_{12}, u_{13}, u_{14}\right\}
$$

$$
\begin{aligned}
& U_{2}=\left\{u_{21}, u_{22}, u_{23}, u_{24}, u_{25}\right\} \\
& U_{3}=\left\{u_{31}, u_{32}, u_{33}\right\} \\
& U_{4}=\left\{u_{41}, u_{42}, u_{43}, u_{44}\right\}
\end{aligned}
$$

By collecting and analyzing data, statistics of four kinds of factors ranked based on importance, has been obtained, see Table 5.

In Table 5, a four-column rank of a matrix has been established in which: sports construction industry is $U_{1}$, sports personnel cultivation is $U_{2}$, sports organizations cultivation is $U_{3}$, sports undertaking revitalization is $U_{4}$.

$$
\begin{aligned}
& U_{2}=\{33,7,4,0\} \\
& U_{2}=\{7,18,18,0\} \\
& U_{3}=\{0,9,23,12\} \\
& U_{4}=\{3,0,19,21\}
\end{aligned}
$$

Weighted vector is obtained from rank 1 to rank 2 $\beta=\left\{\beta_{1}, \beta_{2}, \beta_{3}, \beta_{4}\right\}=\{0.4,0.3,0.2,0.1\}$ 
Table 6. Remarks membership.

\begin{tabular}{|c|c|c|c|c|}
\hline \multirow{2}{*}{ Evaluation Way } & \multicolumn{5}{|c|}{ Set Scores Interval } \\
\cline { 2 - 5 } & $\mathbf{0 - 6 0}$ & $\mathbf{6 0 - 8 0}$ & $\mathbf{8 0 - 9 0}$ & $\mathbf{9 0 - 1 0 0}$ \\
\hline \hline Excellent & 0 & 0 & 0.05 & 0.95 \\
\hline Good & 0 & 0.05 & 0.9 & 0.05 \\
\hline Normal & 0.05 & 0.9 & 0.05 & 0 \\
\hline Bad & 0.95 & 0.05 & 0 & 0 \\
\hline
\end{tabular}

Table 7. China's sports undertaking's evaluation obtained values for all indicators.

\begin{tabular}{|c|c|c|c|}
\hline Sports facilities introduction $u_{11}$ & Excellent & Sports events $u_{31}$ & Excellent \\
\hline Sports facilities maintenance $u_{12}$ & Excellent & Sports activities $u_{32}$ & Good \\
\hline Stadium construction $u_{13}$ & Normal & Sports lecturing $u_{33}$ & Good \\
\hline $\begin{array}{l}\text { Sports equipment maintenance and } \\
\text { changing } u_{15}\end{array}$ & Normal & $\begin{array}{l}\text { International sports undertaking } \\
\text { introduction } u_{41}\end{array}$ & Good \\
\hline $\begin{array}{l}\text { Professional coaches cultivation } \\
\qquad u_{21}\end{array}$ & Excellent & $\begin{array}{l}\text { Sports public undertaking develop- } \\
\text { ment } u_{42}\end{array}$ & Excellent \\
\hline $\begin{array}{l}\text { Sports foreign teachers introduction } \\
\qquad u_{23}\end{array}$ & Excellent & & \\
\hline Training strength $u_{24}$ & Good & & \\
\hline$U_{i}^{*}=U_{i} \cdot \beta^{T}$ & \multicolumn{3}{|c|}{$U_{2}^{*}=\left\{U_{21}, U_{22}, U_{23}, U_{24}\right\}=\{0.53,0.110 .240 .15\}$} \\
\hline$U_{1}^{*}=14, U_{2}^{*}=9.4, U_{3}^{*}=4$ & \multicolumn{3}{|c|}{$U_{1}^{*}=\left\{U_{31}, U_{32}, U_{33}, U_{34}\right\}=\{0.38,0.310 .110 .24\}$} \\
\hline The paper takes normalizatic & \multicolumn{3}{|c|}{$U_{1}^{*}=\left\{U_{41}, U_{42}, U_{43}\right\}=\{0.3,0.40 .3\}$} \\
\hline
\end{tabular}

$U_{1}^{*}=0.35, U_{2}^{*}=0.3, U_{3}^{*}=0.2, U_{4}^{*}=0.15$

It gets

$\bar{A}=\left(\begin{array}{llll}0.35 & 0.3 & 0.2 & 0.15\end{array}\right)$

The paper establishes remarks membership, as shown in Table 6.

China's sport undertaking's evaluation results obtained in sports construction industry, sports personnel cultivation, sports organizations cultivation, and sports undertaking revitalization, are given in Table 7.

By the above model, a single-layer indicator factor fuzzy weight set is:

$$
U_{1}^{*}=\left\{U_{11}, U_{12}, U_{13}, U_{14}, U_{15}\right\}=\{0.26,0.250 .240 .120 .17\}
$$

By analyzing Table $\mathbf{5}$ and Table $\mathbf{3}$, an evaluation set for each column has been established in which: sports construction industry is $U_{1}$, sports personnel cultivation is $U_{2}$, sports organizations cultivation is $U_{3}$, and sports undertaking revitalization is $U_{4}$ :

Sports construction industry $U_{1}=\left(\begin{array}{cccc}0 & 0 & 0.05 & 0.95 \\ 0 & 0 & 0.05 & 0.95 \\ 0 & 0.05 & 0.95 & 0.05 \\ 0 & 0.05 & 0.95 & 0.05 \\ 0 & 0.05 & 0.95 & 0.05\end{array}\right)$

Sports personnel cultivation $U_{2}=\left(\begin{array}{cccc}0 & 0 & 0.05 & 0.95 \\ 0 & 0 & 0.05 & 0.95 \\ 0 & 0 & 0.05 & 0.95 \\ 0 & 0.05 & 0.9 & 0.05\end{array}\right)$ 
Sports organizations cultivation

$U_{3}=\left(\begin{array}{cccc}0 & 0 & 0.05 & 0.95 \\ 0 & 0.05 & 0.9 & 0.05 \\ 0 & 0.05 & 0.9 & 0.05 \\ 0.05 & 0.9 & 0.05 & 0\end{array}\right)$

Sports undertaking revitalization

$U_{4}=\left(\begin{array}{cccc}0 & 0 & 0.05 & 0.95 \\ 0 & 0.05 & 0.9 & 0.05 \\ 0 & 0.05 & 0.9 & 0.05\end{array}\right)$

The above evaluation sets are calculated according to following formula:

$$
B_{i}=A_{i} \cdot R_{i}
$$

The normalization processing is carried out using the obtained $B_{i}$, and thus a fuzzy evaluation matrix is obtained:

$$
\bar{B}=\left(\begin{array}{l}
B_{1} \\
B_{2} \\
B_{3} \\
B_{4}
\end{array}\right)=\left(\begin{array}{cccc}
0.07 & 0.27 & 0.23 & 0.43 \\
0 & 0.1 & 0.7 & 0.5 \\
0.08 & 0.16 & 0.28 & 0.28 \\
0.14 & 0.2 & 0.3 & 0.36
\end{array}\right)
$$

Thus, comprehensive evaluation value is as follows:

$$
Z=U^{*} \cdot B=\left(\begin{array}{llll}
0.40 & 0.05 & 0.34 & 0.18
\end{array}\right)
$$

\section{CONCLUSION}

Sports industry is an industry indispensible for China's economy. This paper conducted status analysis of China's sports industry, and carried out strategic researches on its future development. The paper utilized fuzzy mathematics to analyze recognition of human resources by the outer world, due to suffering from numerous factors, which are fuzzy. The fuzzy mathematics has been used to analyze China government's promotions of sports industry development.

\section{CONFLICT OF INTEREST}

The author confirms that this article content has no conflict of interest.

\section{ACKNOWLEDGEMENTS}

Declared none.

\section{REFERENCES}

[1] Y. Chen, G. Ma, "An empirical study on community sports service residents' Satisfaction Index Model," China Sport Science and Technology, vol. 45, no. 4, 2009.

[2] Y. He, M. Xu, "Study on evaluating system of sports consciousness of community residents in southwest cities," Journal of Chengdu Physical Education Institute, vol. 33, no. 2, pp. 43-45, 2007.

[3] B. Liu, S. Hu, H, Xu, J. Gao, "Indices of the equality of essential public health services in China," Chinese Journal of Health Policy, vol. 2, no. 6, pp. 13-17, 2009.

[4] G. Wang, W. Zhang, "Construction of the evaluation index system of city community sports-taking shanghai as an example," Journal of Chengdu Physical Education Institute, vol. 36, no. 2, 2010.

[5] D. Zhang, M. Li, "Studies on evaluation index system of public sports facilities development level in china," China Sport Science, vol. 33, no. 4, pp. 3-23, 2013.

\footnotetext{
Received: June 10, 2015

Revised: July 29, 2015

Accepted: August 15, 2015

(C) Zhiyong Xu; Licensee Bentham Open.

This is an open access article licensed under the terms of the (https://creativecommons.org/licenses/by/4.0/legalcode), which permits unrestricted, noncommercial use, distribution and reproduction in any medium, provided the work is properly cited.
} 